Artículo

\title{
Litigación oral: estrategia de enseñanza para el desarrollo de competencias argumentativas en la formación de profesores de educación secundaria
}

\author{
Maritza Palma* y María T. Castañeda \\ Universidad del Bío Bío, Avenida Andrés Bello S/N, casilla 447, Chillán, Chile
}

\section{INFORMACIÓN DEL ARTÍCULO}

\section{Historia del artículo:}

Recibido el 30 de mayo de 2014

Aceptado el 22 de octubre de 2014

On-line el 4 de diciembre de 2014

\section{Palabras clave}

Litigación oral

Competencias argumentativas

Formación del profesorado

\section{R E S U M E N}

El objetivo de este artículo es analizar la eficacia de la litigación oral como estrategia de enseñanza para e desarrollo de competencias argumentativas en la formación del profesorado de secundaria. Está basado en los resultados de un estudio de caso exploratorio, realizado con 162 estudiantes que participaron en la implementación de una estrategia, cuyo diseño consideró el aprendizaje basado en problemas, el modelo de argumentación de Toulmin, el enfoque curricular por competencias y el aprendizaje cognitivista y situacionista como fundamentos de la enseñanza contextualizada. Para el desarrollo de la argumentación escrita y oral se consideró la elaboración de pruebas documentales y su presentación en un contexto de simulación de una litigación. Para evaluar la experiencia se realizó un grupo focal con informantes claves, quienes percibieron que la estrategia contribuyó al desarrollo de aprendizajes conceptuales, procedimentales y actitudinales. Se concluye que para el desarrollo de competencias argumentativas en estudiantes de pedagogía resulta clave hacer hincapié en el diseño de estrategias de enseñanza, la utilización y movilización del conocimiento adquirido y la consideración de contextos de adquisición y aplicación preferentemente auténticos o reales.

(C) 2014 Instituto de Ciencias de la Educación de la Universidad de Oviedo. Publicado por Elsevier España, S.L.U. Todos los derechos reservados.

\section{Oral litigation: A teaching strategy for the development of argumentation skills in secondary education teacher training}

\section{A B S T R A C T}

The aim of this paper is to analyze the effectiveness of oral litigation as a teaching strategy for the development of argumentative skills in secondary education teacher training. It is based on the results of an exploratory case study conducted with 162 students who participated in the implementation of a strategy, with a design that took into account problem-based learning, the Toulmin argumentation model, curriculum competence approach, cognitive and situated learning, and contextual foundations of education. For the development of written and oral arguments, presenting documentary evidence was considered in the context of simulating a Litigation. To evaluate the experience, a focus group was conducted with key informants, who felt that the strategy contributed to the development of conceptual, procedural and attitudinal learning. It is concluded that the development of argumentation skills in teacher training students is key in the design of teaching strategies, the use and mobilization of the knowledge acquired, the acquisition contexts, and preferably of authentic or real application.

(c) 2014 Instituto de Ciencias de la Educación de la Universidad de Oviedo. Published by Elsevier España, S.L.U. All rights reserved.

\footnotetext{
* Autor para correspondencia.

Correo electrónico: mpalma@ubiobio.cl (M. Palma).
} 


\section{Introducción}

Los resultados de diversas investigaciones sobre la efectividad de las instituciones formadoras de docentes en Chile (Ávalos, 2003, 2007, 2009; Ávalos y Matus, 2010; Manzi, 2011; Montecinos, 2003; Pedraja, Araneda, Rodríguez y Rodríguez, 2012; Ruffinelli, 2013) coinciden en plantear la existencia de una fuerte dicotomía entre la formación de los profesores y el ejercicio profesional. En este complejo escenario cobran sentido los procesos de reforma curricular que viven las universidades chilenas y la generación de perfiles de egreso basados en competencias genéricas y específicas para la profesión docente. La tarea actual es avanzar en el diseño de estrategias metodológicas que permitan desarrollar y evaluar la formación de dichas competencias en la implementación de los currículos.

Consecuente con lo anterior, en este estudio se propone una estrategia de enseñanza para el desarrollo de competencias argumentativas, implementada con estudiantes de pedagogía para el nivel de educación secundaria, que presentan debilidades tanto en la producción de textos escritos como orales. La formación en competencias argumentativas se considera importante porque forma parte del perfil genérico del docente, consensuado por el proyecto Tuning para Latinoamérica. En el ejercicio de la profesión docente el acto de enseñar es básicamente un proceso comunicativo, en el que los intercambios discursivos son expresados en forma oral, escrita o gestual, tanto con los alumnos, como con otros colegas y padres, permitiendo la interacción.

En este marco de formación de competencias De Longhi et al. (2012) consideran que además de conocer la materia a enseñar, saber preparar actividades, saber dirigir la actividad de los alumnos, saber evaluar y utilizar la investigación e innovación es necesario agregar un nuevo saber específico que se refiere a la capacitación de los futuros docentes en la dimensión comunicativa y dialógica, como proceso mediador en la enseñanza y el aprendizaje. Se trata, según el autor, de "promover discursos coherentes con el conocimiento científico, centrados esencialmente en habilidades cognitivas y lingüísticas de argumentación». Al respecto, Montaño (2013) define la capacidad de comunicación oral y escrita como competencia genérica relevante para el desempeño profesional de los educadores, puesto que «reúne el conjunto de habilidades y destrezas de comprensión y expresión de textos orales y escritos para lograr una comunicación eficiente y eficaz que permita al profesional actuar asertivamente en el ejercicio profesional».

Para el diseño de la estrategia propuesta se ha considerado como marco teórico el modelo de argumentación de Toulmin, Rieke y Janik (1979), la formación de profesores basada en el enfoque de competencias holístico o integrado (Coll, 2007; Gairín, 2011; Montaño, 2013; Pavié, 2011; Perrenoud, 2004); el enfoque de la enseñanza basada en el contexto y los enfoques del aprendizaje cognitivista y situacionista descritos en Caamaño (2011). A estos fundamentos se agrega el aprendizaje basado en problemas (ABP) como técnica didáctica que permite integrar en su diseño los enfoques teóricos estudiados. Específicamente, se ha tomado como base el modelo de ABP $4 \times 4$ diseñado por Prieto et al. (2006) que considera 4 fases en su organización y que se trabaja en 4 contextos diferentes. A continuación se describen estos fundamentos teóricos que sustentaron el diseño.

\section{Modelo de argumentación de Toulmin}

El modelo argumentativo de Toulmin et al. (1979) establece pasos para una argumentación que pueden ser utilizados en cualquier tipo de disciplina o espacio abierto a la disertación o al debate. Crea un modelo de análisis para cualquier tipo de discurso social argumentativo: una conversación, una noticia del periódico, televisión, radio o prensa escrita, una entrevista, en la interacción docente alumno, abogado-cliente, entre otras. Para este modelo, el argumento como estructura compleja se constituye de 6 elementos: una tesis que se va a defender, que debe ser una proposición controversial, clara y desafiante. La tesis se apoya en una evidencia o base que está formada por hechos o condiciones que son observables: estadísticas, citas, reportes, evidencias físicas, que sirven de prueba para la tesis propuesta. Un tercer elemento del argumento lo constituye la garantía que establece la relación entre la base y la tesis. Permite verificar que las bases de la argumentación sean las apropiadas y justificar su importancia. La garantía necesita de un respaldo o apoyo que puede ser un estudio científico, un código, una estadística, o una creencia arraigada dentro de una comunidad. El respaldo es similar a la base, ya que se expresa por medio de estadísticas, testimonios o ejemplos, pero se distingue por apoyar la garantía, mientras que la base apoya la tesis propuesta. El calificador modal especifica el grado de certeza con el que se sostienen los argumentos. La función de un calificador modal es establecer la probabilidad de la tesis planteada, puesto que no es categórica. Se expresan generalmente a través de adverbios: quizá, seguramente, típicamente, usualmente, algunos, pocos, sin duda, probablemente, algunas veces, la mayoría, probablemente, tal vez... Finalmente, completa el modelo de Toulmin el anticipar las refutaciones posibles o contra-argumentaciones, ampliando así las posibilidades de desarrollo argumental de la tesis propuesta, dado que los argumentos no se consideran universalmente verdaderos (Rodríguez, 2004).

\section{La formación de profesores basada en el enfoque por competencias}

En relación con la definición de competencias, se considera como componentes fundamentales del concepto los contenidos de aprendizaje y la importancia del contexto de adquisición y aplicación (Coll, 2007). Se considera un enfoque holístico o integrado, en que competente es la persona que utiliza y moviliza el conocimiento adquirido, integra diferentes tipos de atributos necesarios (conocimientos, habilidades, actitudes y valores) para el desempeño del trabajo en un contexto determinado y de manera exitosa (Pavié, 2011). De esta manera, las competencias movilizan conocimientos, habilidades o actitudes, pertinentes en situación y donde cada situación es única (Perrenoud, 2004).

Gairín (2011) describe el carácter aplicativo y dinámico de las competencias como características que justifican cambios en los diseños de estrategias didácticas y evaluativas orientadas a la formación de competencias en los estudiantes. Según el autor, el diseño de estrategias implica transitar desde un rol docente tradicional, con modelos pedagógicos centrados en la docencia, a un rol docente mediador cuyo modelo pedagógico está centrado en el estudiante. Esto supone un rol activo del estudiante que abandona el rol pasivo, dependiente del profesor y de su planificación. El docente como guía le entrega al estudiante, desde el inicio, información sobre el proceso de aprendizaje, los recursos con que va a contar y los estándares para la evaluación y autoevaluación de su desempeño en la tarea encomendada. Al mismo tiempo, la formación en competencias supone evaluar la capacidad de los estudiantes para articular y movilizar conocimientos, habilidades y actitudes en diversos contextos. Significa, por tanto, evaluar desempeños y establecer niveles de logro de aprendizajes. Implica un predominio de la evaluación diagnóstica, formativa y la metaevaluación (Santos y Moreno, 2004).

En este marco, el desarrollo de competencias supone una forma de trabajo que priorice estrategias pedagógicas para la comprensión profunda, el aprendizaje activo, las relaciones entre saberes y la movilización integrada de conocimientos, habilidades y actitudes en diversos contextos, preferentemente auténticos o reales. Según Montaño (2013) «a través de la estructura académica por competencias se trata de generar espacios de aprendizaje por descubrimiento, experimentación y aplicación, empleo o utilización de 
realidades concretas, así como desarrollar en los estudiantes pensamiento crítico, diálogo, cuestionamiento continuo y reflexión».

Para el desarrollo de competencias la experiencia y la literatura especializada recomiendan integrar diversas metodologías según las necesidades y características del profesorado, de los estudiantes, del contexto epistemológico e institucional, diseñando estrategias metodológicas tan variadas como: síntesis y análisis crítico de libros, presentaciones, mapas conceptuales, resolución de problemas, estudio de casos, trabajos por proyectos, prácticas externas, debates, simulaciones, proyecciones, visitas, entre otras (Gairín, 2011).

\section{El aprendizaje basado en problemas y el desarrollo de competencias en contexto}

En la formación universitaria la resolución de problemas mediante ABP tiene cada vez más aceptación, puesto que permite poner en práctica los postulados del enfoque por competencias. Como metodología ha mostrado su utilidad para que los estudiantes aprendan con profundidad y autonomía, ejercitando y desarrollando competencias genéricas y específicas que necesitará profesionalmente (Prieto et al., 2006). Esta estrategia de enseñanza permite al profesor como didacta y al estudiante como aprendiz integrar aprendizajes cognitivos y situados en un contexto determinado. La tesis principal del aprendizaje situado es que, para que el estudiante aplique lo aprendido, el proceso de enseñanza y aprendizaje debe darse en un contexto auténtico, lo más real posible. En el diseño de estrategias de aprendizaje situado el énfasis debe estar en la situación y el contexto en el cual el aprendizaje tiene lugar (Caamaño, 2011). Por su parte, las teorías cognitivas del aprendizaje consideran que el conocimiento ocurre en la mente del sujeto a partir del desarrollo de procesos cognitivos que se activan cuando este aprende. El conocimiento es considerado como una entidad abstracta que se halla en la mente de los individuos (Caamaño, 2011).

Los planteamientos descritos han servido de sustento teórico para analizar la eficacia de la litigación como estrategia para el desarrollo de competencias argumentativas en la formación del profesorado de secundaria. Se partió del supuesto de que la implementación de una estrategia de litigación oral sería efectiva en el desarrollo de la comunicación oral y escrita en los estudiantes que participaron del estudio.

\section{Método}

\section{Participantes}

Participaron en el estudio un grupo de 162 estudiantes para profesor de educación secundaria, distribuidos en 52 estudiantes de pedagogía en Ciencias Naturales; 31 estudiantes de pedagogía en Historia; 46 estudiantes de pedagogía en Inglés y 33 estudiantes de pedagogía en Castellano. La muestra fue intencionada, siguiendo una estrategia de muestreo comprensible (McMillan y Schumacher, 2005), cuyos criterios de selección se fundamentaron principalmente en los resultados del diagnóstico de una asignatura de primer año del plan de formación genérica, denominada Estrategias de la comunicación. Esta asignatura es común para todas las carreras seleccionadas. A través del diagnóstico de las carreras en estudio fue posible evidenciar de manera transversal un léxico restringido, inadecuada organización de ideas (coherencia), dificultades para fundamentar e integrar planteamientos críticos y por tanto desconocimiento de las superestructuras textuales, por ejemplo, el discurso expositivo y argumentativo. De esta manera, y con la intención de contrastar las posibles diferencias en habilidades comunicativas logradas, dependiendo de la naturaleza pedagógica de las disciplinas estudiadas, se consideraron para la intervención estudiantes regulares de tercer año de formación.

\section{Programa de intervención}

Se diseñó una estrategia que integra tanto la enseñanza contextualizada, el aprendizaje cognitivo, situado y el desarrollo de competencias comunicativas, definidos anteriormente como fundamentos teóricos que respaldan el diseño. Para tal efecto se optó por la técnica didáctica del ABP para organizar la estrategia de aprendizaje completa, y la técnica de debate litigación en juicio oral para trabajar la argumentación. La litigación es una forma específica de debate en que compiten 2 antagonistas que buscan la aprobación de un juez o jurado. Su estructura y etapas resultan de una adaptación al contexto de la sala de clases de los juicios orales en el marco de la reforma al sistema procesal penal chileno (Fuentes, Chávez, Carbonell y Coquelet, 2004).

Se trabajó con el modelo de ABP $4 \times 4$ diseñado por Prieto et al. (2006) que se desarrolla en 4 fases (activación, investigación, resolución y evaluación) distribuidas en 4 escenarios de trabajo diferentes (clase completa, grupo con tutor, grupo sin tutor, trabajo individual) y que por tanto permite trabajar con grupos numerosos de estudiantes. Junto con ello, para construir las argumentaciones necesarias para el juicio oral, se optó por el modelo de Stephen Toulmin. La elección de este modelo se debe a que ofrece un esquema o modelo de argumentación que permite a los estudiantes producir, distinguir, comprender y hasta evaluar discursos argumentativos orales (Fuentes et al., 2004). En la tabla 1 se presenta una síntesis esquemática de la estrategia de enseñanza implementada, abordando 4 fases que coinciden con la estructura de un ABP $4 \times 4$, la estructura argumentativa de Toulmin y en diferentes contextos.

Tabla 1

Estrategia de enseñanza para el desarrollo de competencias argumentativas en la Formación del Profesorado de Secundaria

\begin{tabular}{|c|c|c|c|}
\hline $\begin{array}{l}\text { Litigación oral: } \\
\text { fases de la } \\
\text { argumentación } \\
\text { escrita y oral }\end{array}$ & $\begin{array}{l}\text { Fase ABP } 4 \times 4 \\
\text { (Prieto et al., } \\
2006 \text { ) }\end{array}$ & $\begin{array}{l}\text { Estructura } \\
\text { argumentativa } \\
\text { (Toulmin et al., } \\
\text { 1979) }\end{array}$ & Contextos \\
\hline $\begin{array}{l}\text { 1. Análisis de } \\
\text { cargos (tesis) } \\
\text { planteados en } \\
\text { acta de acusación } \\
\text { para juicio oral }\end{array}$ & $\begin{array}{l}\text { Activación del } \\
\text { conocimiento y } \\
\text { análisis }\end{array}$ & Tesis & Trabajo en aula \\
\hline $\begin{array}{l}\text { 2. Búsqueda de } \\
\text { fundamentos, } \\
\text { razones y base } \\
\text { empírica para las } \\
\text { tesis planteadas } \\
\text { en acta de } \\
\text { acusación } \\
\text { Elaboración de } \\
\text { prueba } \\
\text { documental }\end{array}$ & $\begin{array}{l}\text { Investigación y } \\
\text { estudio }\end{array}$ & $\begin{array}{l}\text { Bases } \\
\text { Garantía } \\
\text { Respaldos }\end{array}$ & $\begin{array}{l}\text { Trabajo } \\
\text { individual } \\
\text { Trabajo grupal } \\
\text { sin tutor }\end{array}$ \\
\hline $\begin{array}{l}\text { 3. Análisis crítico } \\
\text { de } \\
\text { argumentaciones } \\
\text { y anticipación de } \\
\text { contra- } \\
\text { argumentaciones }\end{array}$ & $\begin{array}{l}\text { Resolución del } \\
\text { problema: } \\
\text { consideración } \\
\text { de soluciones e } \\
\text { informe }\end{array}$ & $\begin{array}{l}\text { Calificadores } \\
\text { Modales } \\
\text { Refutaciones } \\
\text { Posibles }\end{array}$ & $\begin{array}{l}\text { Trabajo grupal } \\
\text { con tutor }\end{array}$ \\
\hline $\begin{array}{l}\text { 4. Desarrollo de la } \\
\text { litigación oral } \\
\text { Desarrollo de } \\
\text { metaevaluación }\end{array}$ & $\begin{array}{l}\text { Presentación } \\
\text { ante la clase y } \\
\text { evaluación: } \\
\text { reflexión } \\
\text { metacognitiva }\end{array}$ & $\begin{array}{l}\text { Presentación } \\
\text { de argumentos }\end{array}$ & Trabajo en aula \\
\hline
\end{tabular}

Síntesis esquemática de la estrategia de enseñanza implementada, abordando 4 fases que coinciden con la estructura de un $\mathrm{ABP} 4 \times 4$, la estructura argumentativa de Toulmin y en diferentes contextos. 


\section{Procedimiento}

El estudio se desarrolla en el transcurso del segundo semestre de 2013, en el marco de una asignatura de la formación pedagógica ubicada en el tercer año del plan de estudios. Para llevar a cabo la implementación de la estrategia el equipo de investigación capacitó al profesor a cargo de la asignatura, quien la desarrolló aproximadamente durante un mes de clases regulares, en todas sus fases. Tanto los materiales utilizados como la logística aplicada en su desarrollo fueron diseñados y producidos por los investigadores en el marco de un proyecto de investigación regular financiado con recursos institucionales (Código 124723 3/R).

El procedimiento consideró la implementación de la estrategia en 4 fases que se describen a continuación:

1. Activación del conocimiento y análisis: análisis de cargos planteados en acta de acusación para litigación oral. La estrategia didáctica se desarrolló a lo largo de 4 semanas, siguiendo el esquema de fases del ABP $4 \times 4$. La primera fase se desarrolló considerando la clase completa de estudiantes de las 4 carreras, y durante ella recibieron el entrenamiento metodológico inicial, la descripción de los desempeños de competencias que serían evaluadas y las normas e instrucciones claras para el desarrollo del trabajo individual y sin tutoría que conformará las siguientes etapas del ABP. En esta fase se presentó el problema, consistente en una acusación de 17 cargos en contra de la evaluación educacional. Se plantea que el acusado merece un juicio justo y, por lo tanto, se solicita al curso completo preparar la litigación.

En cada carrera participante se formaron 2 grupos de igual número de estudiantes que mediante sorteo asumieron la fiscalía o la defensa del acusado, respectivamente. En el interior de cada equipo se repartieron, según habilidades y destrezas, los roles de abogado, procuradores y testigos para conformar el equipo de debate para la litigación oral. Una vez conformados los equipos de trabajo procedieron al análisis de los 17 cargos presentados en el "acta de acusación» para identificar elementos del problema y dar cumplimiento así a la primera etapa en el diseño de la estructura argumental.

2. Investigación y estudio: búsqueda de fundamentos, razones y base empírica para las tesis planteadas en acta de acusación. En esta fase los equipos denominados "fiscalía» $y$ «defensa» respectivamente se abocaron a la tarea de indagar los fundamentos, razones y base empírica de las tesis (cargos) planteadas en acta de acusación. Debieron investigar para responder las siguientes interrogantes implicadas en el diseño de la estrategia argumentativa para ganar el juicio: primero, ¿cuál es la información desde donde parten estas tesis/cargos?; segundo, ¿qué estamos garantizando con las bases que hemos entregado para sostener estas tesis/cargos?; y por último, ¿con qué información respaldaremos nuestra tesis? Esta etapa se desarrolló en contextos de trabajo individual y grupal sin tutor, además de considerar horas de trabajo extraaula. Se estructuró en 2 etapas: primero de investigación individual y luego de puesta en común de lo investigado. Este ciclo podía repetirse tantas veces como fuera necesario, dada la naturaleza de la problemática estudiada. Para el desarrollo de la investigación se utilizaron distintas fuentes de información, fundamentalmente las fuentes académicas tanto impresas como en formato virtual. El producto final de esta fase lo constituye la prueba documental elaborada.

3. Resolución del problema: análisis crítico de argumentaciones $y$ anticipación de contra-argumentaciones. En esta etapa cada equipo resolvió y redactó la estructura argumental definitiva para ganar el juicio oral, constituido por el alegato de apertura y los argumentos de los testigos como medios de prueba. Previo a esto, reunidos en los grupos de trabajo y bajo la tutoría del profesor, realizaron análisis crítico de las argumentaciones construidas hasta ahora, anticipando las posibles contraargumentaciones o refutaciones del equipo contendor. Para evitar que fueran conocidas las estrategias argumentales por el equipo contrario antes del juicio oral, el profesor debió asesorar por separado a la "fiscalía» y a la "defensa».

4. Presentación ante la clase y evaluación: desarrollo de la litigación oral. Esta fase contempló 2 etapas: a) la sesión de simulación de la litigación oral; y la b) metaevaluación, sesión de evaluación de la actividad. Respecto de la primera, una vez cumplida la fecha dispuesta al inicio de la estrategia para la realización del debate, se procedió a recrear el contexto de un juicio oral, lo más auténtico posible. Como escenario del debate se simuló una sala de tribunal en que participaron un abogado en ejercicio representando el rol de juez, un secretario de actas y el grupo de debate representado por los estudiantes de cada carrera participante, ejerciendo roles de abogado defensor, fiscal, procuradores y testigos. La sesión de debate se desarrolló bajo el estricto rigor de una asamblea de tribunal basado en el Código penal chileno, con un reglamento para el uso de la palabra que estructuró los tiempos para el alegato de apertura, el interrogatorio de los testigos y la presentación del alegato final. Una vez expuestos los alegatos finales, el juez debió deliberar y llegar a un veredicto: la evaluación educacional es culpable o inocente de los cargos que se le imputan. Una vez emitido el veredicto, el juez debió en conciencia dictar sentencia.

Terminada la sesión de debate, en una segunda etapa de esta fase, el profesor dirigió la discusión y reflexión grupal acerca de la estrategia de enseñanza implementada, los aprendizajes desarrollados con ella, las fortalezas y debilidades detectadas como categorías de análisis crítico metacognitivo. Posteriormente, el profesor junto a los estudiantes que desempeñaron roles protagónicos durante el ABP, evaluaron el desempeño logrado mediante una rúbrica de evaluación para juicio oral, conocida y socializada al inicio del ABP con los estudiantes participantes.

\section{Métodos e instrumentos de evaluación}

Se siguió una metodología de corte cualitativo. El diseño de la investigación fue un estudio de caso exploratorio, delineado con el fin de explorar la eficacia de la estrategia de enseñanza implementada con estudiantes para profesor de educación secundaria en una universidad tradicional chilena (McMillan y Schumacher, 2005). El estudio involucró las siguientes 3 estrategias de recolección de datos coincidentes con las fases del proceso de implementación de la estrategia de enseñanza aplicada:

1. Primera fase: evaluación formativa de la prueba documental. Se evaluaron los argumentos y contra-argumentos elaborados individualmente por los estudiantes, a través de la revisión de informes escritos de la prueba documental. A través de un análisis inductivo se identificaron fortalezas y debilidades de la producción escrita. Para realizar este análisis se consideró como unidad de estudio, de carácter intencionado, los documentos elaborados por los estudiantes como prueba documental, los que fueron interpretados a través de la técnica de análisis de contenidos (Valles, 1999). Específicamente, el análisis de la información se desarrolló a partir de la contrastación de categorías teóricas provenientes del modelo argumentativo de Toulmin et al. (1979).

2. Segunda fase: evaluación del nivel de desarrollo de las competencias argumentativas. Se aplicó una rúbrica holística, como instrumento que permitió hacer una apreciación mediante una escala de 3 niveles: alto, medio y bajo nivel de desarrollo de la competencia. El instrumento fue diseñado considerando la 
descripción de los niveles de desarrollo de la competencia de comunicación oral y escrita según Montaño (2013).

3. Tercera fase: evaluación de la experiencia de enseñanza aprendizaje vivenciada. Se realizó un grupo focal con informantes clave de cada carrera. La selección de informantes clave se realizó teniendo en cuenta los resultados de las evaluaciones descritas anteriormente, es decir, fueron incluidos en el grupo estudiantes con buen y mal desempeño en la producción de argumentos escritos, estudiantes que obtuvieron un nivel alto, medio y bajo en el desarrollo de la competencia argumentativa al aplicar la rúbrica respectiva, y como último criterio de selección se consideró a los estudiantes que desempeñaron los roles más activos durante la litigación oral, tales como el defensor, el fiscal y un testigo por cada equipo. La sesión de evaluación de la experiencia fue grabada y posteriormente se procedió a la transcripción literal de las marcas lingüísticas y paralingüísticas para realizar un análisis de contenido contextualizado (Gordo y Serrano, 2008).

\section{Resultados}

Fortalezas y debilidades detectadas en la producción de argumentos escritos

Las fortalezas de los estudiantes en la producción de argumentos, que emergen del análisis realizado a sus producciones escritas, establecen relación con la extracción de información de diversas fuentes y su utilización pertinente al redactar textos. La mayor parte de los estudiantes reconocen, saben ubicar y extraer información de diversos tipos de material impreso y virtual como base de sus escritos. Además, exponen sus ideas con coherencia y corrección. La argumentación se apoya, por tanto, en una base y en un respaldo formado por un estudio científico, un código, una estadística, o una creencia arraigada que, a su vez, sirven de prueba para la tesis y la garantía. Esto se evidencia en la citación de autores y de resultados de investigaciones, lo que da mayor validez a sus escritos. Lo anterior da cuenta de que los estudiantes identifican y distinguen un dato de un supuesto.

Por su parte, las principales debilidades que emergen del análisis realizado a la prueba documental elaborada por los estudiantes, establecen relación con la utilización de calificadores modales y de refutaciones posibles. La mayor parte de los estudiantes no establecen el grado de certeza de las tesis planteadas, lo que evidencia poca profundidad de la argumentación. Es decir, la investigación realizada para elaborar argumentos es insuficiente. Esto queda en evidencia también, en el débil planteamiento de contra-argumentaciones. En la argumentación elaborada no se ha considerado al destinatario para seleccionar los argumentos más adecuados y eficaces, $\mathrm{y}$ para exponer razones que contrarresten o invaliden los razonamientos ajenos. Esto último limita las posibilidades de desarrollo argumental de la tesis propuesta.

\section{Nivel de desarrollo de competencias argumentativas}

Para establecer el nivel de desarrollo de las competencias argumentativas se utilizó una matriz de valoración de 3 niveles (bajo, medio y alto) diseñada para evaluar el desempeño mostrado por los estudiantes en relación con la comunicación oral y escrita. La primera fue evaluada durante el desarrollo de la litigación oral y la segunda durante el proceso de elaboración de la prueba documental (tablas 2 y 3 ).

En la tabla 2 se observa que la mayoría de los estudiantes evaluados obtiene un nivel de logro medio en la comunicación oral. Esto significa que un 63\% de ellos muestra capacidad de escucha y se expresa oralmente con espontaneidad, seguridad y pertinencia. Usan una entonación adecuada, dando expresividad a sus mensajes verbales, influyendo en la atención de los oyentes. Producen mensajes verbales claros en los que se evidencia dominio de recursos expresivos con sentido lógico. Ordenan y dan coherencia a la exposición de sus ideas, revelando posesión de información académica. Exponen, por tanto, con seguridad y pertinencia mensajes orales académicos.

Destaca la carrera de pedagogía en Inglés, con un 26\% de estudiantes que alcanza un nivel de logro alto para la competencia de comunicación oral. Esto significa que poseen habilidades lingüísticas comunicativas desarrolladas para un mejor desempeño personal y académico, lo que se revela en su adecuada capacidad de escucha y su eficiente y eficaz comunicación verbal.

Por el contrario, pedagogía en Castellano registra un 27\% de estudiantes con un nivel de logro bajo en la comunicación oral, lo que significa que expresan sus ideas de forma verbal con claridad y persuasión, evidencian tener capacidad de escucha, tolerancia y respeto en los contextos comunicativos en que interactúan lingüísticamente, sin embargo, aún no poseen habilidades lingüísticas comunicativas desarrolladas para un mejor desempeño personal y académico.

La tabla 3 presenta los resultados obtenidos por los estudiantes en relación con la comunicación escrita.

En la tabla 3 se observa que un 94\% de los estudiantes participantes del estudio se encuentran en los niveles medio y alto de desempeño en comunicación escrita. Un $57 \%$ de ellos evidencia condiciones para reconocer, ubicar y extraer información de diversos

Tabla 2

Rúbrica para evaluar comunicación oral

\begin{tabular}{|c|c|c|c|c|}
\hline Carrera & Nivel bajo & Nivel medio & Nivel alto & $\begin{array}{l}\text { Número total } \\
\text { de alumnos } \\
\text { por carrera }\end{array}$ \\
\hline & $\begin{array}{l}\text { Expresa sus ideas de forma } \\
\text { verbal con claridad y } \\
\text { persuasión, evidenciando tener } \\
\text { capacidad de escuchar, tolerar } \\
\text { y respetar en los contextos } \\
\text { comunicativos en que } \\
\text { interactúa lingüísticamente }\end{array}$ & $\begin{array}{l}\text { Se comunica de forma oral con } \\
\text { claridad, fluidez y naturalidad } \\
\text { expresando sus ideas con } \\
\text { sentido lógico y pensamiento } \\
\text { reflexivo en situaciones } \\
\text { comunicativas académicas } \\
\text { con incidencia en la utilización } \\
\text { del lenguaje llano y las } \\
\text { convenciones del diálogo }\end{array}$ & $\begin{array}{l}\text { Posee habilidades lingüísticas } \\
\text { comunicativas desarrolladas } \\
\text { para un mejor desempeño } \\
\text { personal y académico, lo que se } \\
\text { revela en su adecuada } \\
\text { capacidad de escucha y su } \\
\text { eficiente y eficaz comunicación } \\
\text { verbal }\end{array}$ & \\
\hline Pedagogía en Historia & $17 \%$ & $67 \%$ & $17 \%$ & 31 \\
\hline Pedagogía en Castellano & $27 \%$ & $55 \%$ & $18 \%$ & 33 \\
\hline Pedagogía en Ciencias & $6 \%$ & $71 \%$ & $23 \%$ & 52 \\
\hline Pedagogía en Inglés & $15 \%$ & $59 \%$ & $26 \%$ & 46 \\
\hline Totales & $15 \%$ & $63 \%$ & $22 \%$ & 162 \\
\hline
\end{tabular}

Evaluación de los niveles de desempeño en comunicación oral en estudiantes de la formación inicial docente. 
Tabla 3

Rúbrica para evaluar comunicación escrita

\begin{tabular}{|c|c|c|c|c|}
\hline Carrera & Nivel bajo & Nivel medio & Nivel alto & $\begin{array}{l}\text { Número total } \\
\text { alumnos } \\
\text { por carrera }\end{array}$ \\
\hline & $\begin{array}{l}\text { Produce textos escritos de } \\
\text { distinto tipo relacionados } \\
\text { con sus experiencias de } \\
\text { aprendizaje, con sentido lógico } \\
\text { y pensamiento reflexivo } \\
\text { aplicando la normativa } \\
\text { gramatical y ortográfica } \\
\text { vigente }\end{array}$ & $\begin{array}{l}\text { Redacta textos informativos, } \\
\text { argumentativos y descriptivos } \\
\text { aplicando la normativa } \\
\text { gramatical y ortográfica } \\
\text { vigente para expresar con } \\
\text { coherencia, claridad y solidez } \\
\text { sus conocimientos }\end{array}$ & $\begin{array}{l}\text { Construye textos escritos } \\
\text { de alta calidad expresando } \\
\text { con coherencia, solidez } \\
\text { y propiedad sus ideas, } \\
\text { experiencias y argumentos } \\
\text { utilizando las convenciones de } \\
\text { la estructura y presentación de } \\
\text { textos académicos y científicos }\end{array}$ & \\
\hline Pedagogía en Historia & $3 \%$ & $40 \%$ & $57 \%$ & 31 \\
\hline Pedagogía en Castellano & $3 \%$ & $24 \%$ & $73 \%$ & 33 \\
\hline Pedagogía en Ciencias & $0 \%$ & $38 \%$ & $62 \%$ & 52 \\
\hline Pedagogía en Inglés & $17 \%$ & $41 \%$ & $41 \%$ & 46 \\
\hline Totales & $6 \%$ & $37 \%$ & $57 \%$ & 162 \\
\hline
\end{tabular}

Evaluación de los niveles de desempeño en comunicación escrita en estudiantes de la formación inicial docente.

tipos de material impreso, redactar textos de divulgación general, manejar textos de comunicación académica, informativa y argumentativa, redactar textos académicos en los que exponen sus ideas con coherencia y corrección y producir textos científicos que consideren las convenciones de redacción y presentación. La carrera de pedagogía en Ciencias Naturales presenta más altos niveles de logro, con $62 \%$ y $38 \%$ de estudiantes con niveles de desarrollo alto y medio respectivamente, no registrando estudiantes con niveles de logro bajo.

Similares resultados obtiene la carrera de pedagogía en Castellano, con un $73 \%$ de estudiantes que alcanza un nivel de logro alto para la competencia de comunicación escrita. Esto significa que usan reflexivamente el lenguaje para la generación de textos escritos académicos y científicos de calidad. Este resultado llama la atención si se considera que el $27 \%$ de los estudiantes de esta carrera obtuvo un nivel de logro bajo en la comunicación oral. Por su parte, en la carrera de pedagogía en Inglés un 17\% de los estudiantes obtiene un nivel de logro bajo en comunicación escrita, en contraste con un $26 \%$ de los estudiantes de esta carrera que alcanza un nivel de logro alto para la comunicación oral.

\section{Percepción de los estudiantes en relación con la experiencia de enseñanza-aprendizaje vivenciada}

Del análisis cualitativo de las respuestas y comentarios realizados por los estudiantes durante el desarrollo del grupo focal, emergen como principales categorías los aprendizajes desarrollados, las debilidades percibidas y las percepciones sobre su aplicación en la práctica docente.

Respecto de los aprendizajes desarrollados, los estudiantes consideran que la estrategia de enseñanza experimentada les permitió profundizar sus conocimientos factuales y conceptuales junto con desarrollar habilidades y actitudes. Señalan que la estrategia favoreció el trabajo colaborativo, la búsqueda de información de manera autónoma y autorregulada. Manifiestan que el simular un contexto auténtico de litigación oral, con presencia de un abogado de profesión en el rol de juez, les condicionó al desempeño eficiente y eficaz de la comunicación oral. Por su parte, reconocen que el modelo de Toulmin aplicado favoreció la argumentación escrita, al presentarles una estructura que guiaba el trabajo de elaboración de la prueba argumental.

A partir de los comentarios y opiniones expresadas por los estudiantes es posible interpretar también que la estrategia aplicada favoreció procesos metacognitivos respecto de los aprendizajes logrados y las actividades desarrolladas: «con el juicio oral uno se da cuenta de que con una buena argumentación uno puede tener la capacidad de convencimiento que nosotros como futuros profesores necesitaremos», "aprendí cómo se realiza un buen argumento, pude poner en práctica todo lo que aprendí durante las clases»; "aprendí que no solo los buenos argumentos son los que triunfan, la performance al momento de exponer es un factor predominante», "aprendí la importancia de la comunicación oral, el trabajo en equipo y la selección de información».

Sumado a lo anterior, los estudiantes perciben que la estrategia vivenciada favoreció el desarrollo de las habilidades comunicativas, tanto orales como escritas, además de habilidades de investigación, argumentación y contra-argumentación: «la habilidad más importante fue la búsqueda de información basada en fuentes para estructurar la acusación", "pude mejorar mis habilidades comunicativas, de trabajo en equipo, de investigación» «la estrategia me permitió desarrollar la argumentación, la comunicación oral de ideas y el trabajo en equipo»; «dentro de las habilidades desarrollé la investigativa al buscar más contra-argumentos que argumentos para mi prueba documental».

Resulta interesante destacar que para algunos estudiantes la estrategia les permitió descubrir en sí mismos habilidades que les resultaban desconocidas: "tener un alto cargo en este juicio me fue muy útil para desarrollar habilidades que ni siquiera sabía que poseía, como las habilidades sociales y de liderazgo. Agradezco enormemente esta oportunidad», "aprendí que soy capaz de utilizar el inglés de manera investigativa y en profundidad, y también soy capaz de integrar nuevos conceptos técnicos"; "gracias a esta estrategia he podido leer más comprensivamente, hablar con fundamentos, buscar información con mayor eficacia»; "actividades como el juicio oral son muy útiles, porque nos pone en una situación real, lo que requiere de una mejor preparación además de una instancia para poder poner en práctica nuestras habilidades comunicativas».

Los estudiantes consideran que con la estrategia vivida se potencia el trabajo en equipo, al permitir el desarrollo de actitudes como la empatía, la perseverancia, la responsabilidad, la tolerancia, la disposición al trabajo, la tolerancia a la frustración, la motivación y la sana competencia: "se trabajó el respeto, la empatía, pensar en la sobrecarga del defensor y ayudarlo, ser parte de un equipo, entre otras»; "Pude observar que mi grupo fue muy unido, muy dispuesto, y cabe destacar que siempre hubo un grupo que trabajó más que otros, pero gracias al juicio oral pude ser menos introvertido, pude observar mucho compañerismo y aprendí a ser más responsable»; "respeto, saber esperar, tener mucha paciencia con los que trabajan poco, siempre puede ser mejor, no conformarse».

El planteamiento de los estudiantes respecto de las debilidades de la estrategia vivida está en relación con el tiempo para el desarrollo de la estrategia, la conformación numerosa de los equipos de trabajo, sumado a sus aprehensiones respecto de la parcialidad del juez y las estrategias de evaluación utilizadas. Consideran una debilidad lo numeroso de los grupos de trabajo, 
ya que dificultó la organización y la participación de todos. Estas dificultades las atribuyen directamente a la organización de la estrategia más que a sus propias habilidades de trabajo en equipo. Llama la atención también que perciban como debilidad la ausencia de autoevaluación individual y grupal.

En los relatos de los estudiantes es posible observar contradicciones entre lo que consideraron como fortalezas de la estrategia y luego aquello que percibieron como debilidades. Por ejemplo, plantean que la distribución y coordinación del trabajo y los roles dentro del grupo fue ineficiente, indican que faltó mayor monitorización, señalan que la estrategia fomentaba la competencia, y por tanto hubo discriminación entre los compañeros de equipo al dejar excluidos a quienes poseían menos habilidades comunicativas. Surgen también críticas a la estrategia por considerar que "fomenta la flojera», "se pierde tiempo» y porque permite que solo algunos participen.

Sobre la factibilidad de aplicar la estrategia experimentada en la propia práctica docente, los estudiantes manifiestan nuevamente sus creencias respecto de la práctica de un profesor. Señalan que se trata de una estrategia muy compleja como para ser aplicada en un contexto real de la educación primaria y secundaria. No se sienten capaces de implementarla, lo que evidencia creencias sobre sí mismos, es decir sobre su autoeficacia.

Finalmente, aun cuando valoraron positivamente la experiencia, la consideran innovadora, motivadora, favorecedora de la colaboración, promotora de la investigación, de la aplicación y del desarrollo de habilidades sociales y comunicativas, se muestran escépticos sobre la factibilidad de su aplicación en contextos distintos al universitario, por razones de tiempo, por las características de la gestión de los establecimientos educacionales y por las características propias de los estudiantes de primaria y secundaria.

\section{Discusión}

La simulación de una litigación oral es utilizada en Chile para entrenar a los estudiantes de derecho en las destrezas necesarias para ejercer como jueces, fiscales, defensores y abogados particulares en el marco de la reforma procesal penal chilena, por lo tanto, se ha comprobado su efectividad en el desarrollo de competencias argumentativas en la formación de dichos profesionales (Fuentes et al., 2004). Sin embargo, no existen estudios publicados acerca de la efectividad de la litigación con estudiantes de pedagogía, y a pesar de que aún se trata de un estudio exploratorio, se concluye que la litigación oral, utilizada como estrategia de enseñanza, favorece el desarrollo de competencias argumentativas en la formación inicial de profesores.

A partir de lo anterior se advierte que para el desarrollo de competencias en estudiantes de educación superior resultan elementos claves a considerar en el diseño de estrategias de enseñanza la utilización y movilización del conocimiento adquirido, la integración de diferentes tipos de contenidos de aprendizaje y la importancia del contexto de adquisición y aplicación. Estos hallazgos son coincidentes con lo señalado por Perrenoud (2004); Coll (2007); Pavié (2011); Gairín (2011) y Montaño (2013) respecto de la formación de profesores basada en el desarrollo de competencias. De esta forma, las estrategias de enseñanza utilizadas en la formación inicial de profesores responderán a las necesidades y demandas que genera el ejercicio de la profesión, incidiendo en las prácticas en el aula de los futuros docentes y con ello los aprendizajes de sus estudiantes, coincidiendo con los planteamientos de Ávalos (2003, 2007, 2009); Ávalos y Matus (2010); Manzi (2011); Montecinos (2003); Pedraja et al. (2012) y Ruffinelli (2013) respecto de la formación de profesionales de la educación.

La propuesta de enseñanza diseñada favoreció el desarrollo de competencias argumentativas en estudiantes de las 4 carreras de pedagogía participantes en el estudio. Al respecto, son los propios estudiantes los que confirman este hallazgo al manifestar su percepción positiva respecto del desarrollo de sus habilidades sociales y comunicativas debido a la estrategia experimentada. Estos resultados corroboran la propuesta de De Longhi et al. (2012) sobre la necesidad de promover en la formación inicial de los profesores discursos coherentes con el conocimiento científico, centrados esencialmente en habilidades cognitivas y lingüísticas de argumentación.

Fundamentalmente, la presentación de argumentos en el contexto de una litigación oral, con participación de un abogado de profesión que aportó realismo y autenticidad a la estrategia, motivó a los estudiantes a encontrar las evidencias que fundamentaron las tesis planteadas en la prueba documental. Esta etapa de elaboración escrita de argumentos complementó la fase de exposición oral. Aquí el diseño intencionado de la enseñanza contextualizada, que integró tanto aprendizajes cognitivos como situados, fue clave. Se corrobora la tesis principal del aprendizaje situado: el proceso de enseñanza y aprendizaje debe darse en un contexto auténtico, lo más real posible, para que el estudiante aplique lo aprendido (Caamaño, 2011). Por lo tanto, en el diseño de estrategias de aprendizaje situado, el énfasis debe estar en la situación y el contexto en el cual el aprendizaje tiene lugar.

La aplicación de estrategias de resolución de problemas presenta dificultades cuando se trabaja con grupos numerosos de estudiantes. Esta desventaja no permite su desarrollo permanente, sino solo como un evento durante un curso. De esta forma, la vivencia de la estrategia en una sola ocasión no es suficiente para que los estudiantes tomen conciencia (metacognición) de su aporte en el desarrollo de aprendizajes como competencias básicas para su desempeño profesional futuro. Por lo tanto, tal como lo sugiere Prieto et al. (2006), el ABP como metodología ha mostrado su utilidad para que los estudiantes ejerciten y desarrollen competencias genéricas y específicas, por lo que puede ser un modelo utilizado transversalmente en el curriculum de la formación inicial, como una estrategia dinámica que incorpore aquellos elementos ausentes en la enseñanza tradicional.

Las prácticas tradicionales de enseñanza en la formación de profesores, es decir, el predominio de la clase expositiva y en consecuencia el rol pasivo del estudiante, explican las dificultades percibidas por los participantes, tanto en la puesta en práctica de sus habilidades sociales y comunicativas como en la factibilidad de replicar la experiencia en sus futuras prácticas docentes. Los estudiantes perciben una mayor efectividad de la clase tradicional expositiva sobre las estrategias activo-participativas en relación, por ejemplo, con el uso del tiempo. A esto se agrega que en el desarrollo de estrategias de enseñanza que implican colaboración enfrentan dificultades propias de cualquier equipo acostumbrado a la clase frontal y al trabajo en grupo pequeño. Hace falta, por tanto, vivenciar más estrategias activo-participativas para potenciar el reconocimiento de las propias creencias acerca de la enseñanza y el análisis crítico de las mismas que posibilite un cambio. Los resultados en el nivel de desarrollo de competencia comunicativa, tanto oral como escrita, podrían explicarse preliminarmente debido a una práctica formativa tradicional que tendrá incidencia en la efectividad de los futuros profesores para lograr aprendizajes en sus estudiantes, coincidiendo con los planteamientos de Ávalos (2003, 2007, 2009); Ávalos y Matus (2010); Manzi (2011); Montecinos (2003); Pedraja et al. (2012) y Ruffinelli (2013) sobre la formación inicial docente en Chile.

Como caso de estudio exploratorio hubo una serie de limitaciones que se describen explícitamente para que los lectores puedan interpretar nuestros resultados con un adecuado grado de escepticismo. Por tratarse de un estudio exploratorio cualquier generalización de las conclusiones derivadas de este estudio a otros contextos debería hacerse con precaución. Una investigación 
futura requerirá de un diseño cuasi-experimental que permita cautelar la intervención de variables contaminadoras que pudieron afectar el estudio sobre la eficacia de la estrategia implementada en el desarrollo de competencias argumentativas. Además, el tratamiento estadístico de los datos recolectados en un diseño cuasi-experimental permitirá establecer si existen diferencias significativas entre las carreras estudiadas y entre las mediciones que podrían realizarse pre y postintervención comparando un grupo control y uno experimental. A pesar de estas limitaciones, los resultados de este estudio de caso exploratorio son alentadores respecto de la eficacia de la litigación como potencial estrategia para el desarrollo de competencias genéricas en la formación de profesores.

\section{Financiación}

Financiación de la Dirección de Investigación de la Universidad del Bío-Bío, Chile.

\section{Conflicto de intereses}

Los autores declaran no tener ningún conflicto de intereses.

\section{Agradecimientos}

Este trabajo forma parte del Proyecto de Investigación Regular denominado «Estrategia de enseñanza a través de la resolución de problemas según Stephen Toulmin y grupos cooperativos, como herramienta de mejoramiento de la formación inicial docente». Código 124723 3/R.

\section{Referencias bibliográficas}

Ávalos, B. (2003). La formación docente inicial en Chile. Informe final Programa de Fortalecimiento de la Formación Inicial Docente. Unesco.

Ávalos, B. (2007). El desarrollo profesional continuo de los docentes: lo que nos dice la experiencia internacional y de la región latinoamericana. Revista Pensamiento Educativo, 41, 77-99.
Ávalos, B. (2009). La inserción profesional de los docentes. Revista de Curriculum y Formación del Profesorado, 13, 43-59.

Ávalos, B.y Matus, C.(2010). La formación inicial docente en chile desde una perspectiva internacional: informe nacional del estudio internacional IEA TEDS M, Santiago. Ministerio de Educación de Chile.

Caamaño, A. (2011). Enseñar química mediante la contextualización, la indagación y la modelización. Alambique. Didáctica de las Ciencias Experimentales, 17, 21-34.

Coll, C. (2007). Las competencias en la educación escolar: algo más que una moda y mucho menos que un remedio. Aula de Innovación Educativa, 161, 34-39.

De Longhi, A., Ferreyra, A., Peme, C., Bermudez, G., Quse, L., Martínez, S., et al. (2012) La interacción comunicativa en clases de ciencias naturales. Un análisis didáctico a través de circuitos discursivos. Revista Eureka sobre Enseñanza y Divulgación de las Ciencias, 9, 178-195.

Fuentes, C., Chávez, P., Carbonell, V. y Coquelet, J. (2004). Debates estudiantiles, manual de apoyo a la docencia. Chile: Ministerio de Educación.

Gairín, J. (2011). Formación de profesores basada en competencias. Bordón, 63, 93-108.

Gordo A, Serrano A, coordinadores. Estrategias y prácticas cualitativas de investigación social, Madrid: Pearson Prentice Hall; 2008.

Manzi, J. (2011). ¿Qué características de la formación inicial de los docentes se asocian a mayores avances en su aprendizaje de conocimientos disciplinarios? Chile: Proyecto FONIDE N. : F511015.

McMillan, J. y Schumacher, S. (2005). Investigación educativa. Una introducción conceptual (5. a edición.). Madrid: Ed. Pearson Prentice Hall.

Montaño, A. (2013). Educación superior en América Latina: reflexiones y perspectivas en educación. Bilbao: Publicaciones de la Universidad de Deusto.

Montecinos, C. (2003). Desarrollo profesional docente y aprendizaje colectivo. Psicoperspectivas, 2, 105-128.

Pavié, A. (2011). Formación docente: hacia una definición del concepto de competencia profesional docente. Revista Electrónica Interuniversitaria de Formación del Profesorado, 14, 67-80.

Perrenoud, P. (2004). Desarrollar la práctica reflexiva en el oficio de enseñar: profesionalización y razón pedagógica. Barcelona: Graó.

Pedraja, L., Araneda, C., Rodríguez, E. y Rodríguez, J. (2012). Calidad en la formación inicial docente: evidencia empírica en las universidades chilenas. Formación Universitaria, 5, 15-26.

Prieto, A., Barbarroja, J., Reyes, E., Monserrat, J., Díaz, D., Villarroel, M., et al. (2006). Un nuevo modelo de aprendizaje basado en problemas, el ABP $4 \times 4$, es eficaz para desarrollar competencias profesionales valiosas en asignaturas con más de 100 alumnos. Aula Abierta, 87, 171-194.

Rodríguez, L. (2004). El modelo argumentativo de Toulmin en la escritura de artículos de investigación educativa. Revista Digital Universitaria, 5, 2-18.

Ruffinelli, A. (2013). La calidad de la formación inicial docente en Chile: la perspectiva de los profesores principiantes. Calidad en la Educación, 39, 117-154.

Santos, M. y Moreno, T. (2004). ¿El momento de la metaevaluación educativa? Revista Mexicana de Investigación Educativa, 9, 913-931.

Toulmin, S., Rieke, T. y Janik, A. (1979). An introduction to reasoning. New York: Macmillan.

Valles, M. (1999). Técnicas cualitativas de investigación social. Madrid: Síntesis. 